\title{
Understanding Jordanian Mothers' Experience after Stillbirth: A Qualitative Study Protocol
}

\author{
Sahar Mohammed Al-Shuqerat ${ }^{1 *}$ (1), Halah Ahmad Bawadi ${ }^{2 *}$ \\ ${ }^{1}$ School of Nursing, University of Jordan, Amman, Jordan \\ ${ }^{2}$ Maternal and Child Health Nursing Department, School of Nursing, The University of Jordan, Amman, Jordan \\ Email: ^sm_shs14yahoo.com, *h.bawadi@ju.edu.jo
}

How to cite this paper: Al-Shuqerat, S.M and Bawadi, H.A. (2020) Understanding Jordanian Mothers' Experience after Stillbirth: A Qualitative Study Protocol. Open Journal of Nursing, 10, 277-285. https://doi.org/10.4236/ojn.2020.103019

Received: February 17, 2020

Accepted: March 22, 2020

Published: March 25, 2020

Copyright $\odot 2020$ by author(s) and Scientific Research Publishing Inc. This work is licensed under the Creative Commons Attribution International License (CC BY 4.0).

http://creativecommons.org/licenses/by/4.0/

(c) (i) Open Access

\begin{abstract}
Background: Low and middle income countries have the highest rates of stillbirths in the world. In Jordan, stillbirth and how it impacts on mothers who experience it is under-researched. The stories of mothers who have had a stillbirth need to be told and the silence associated with stillbirth needs to be broken. The aim of this study is to explore and understand the meanings attributed by mothers' to their experience of stillbirth in Jordan. Methods: The study will employ phenomenological qualitative research design. Semi-structured interview will be conducted with the mothers' who will be recruited from three governmental hospitals in the north, middle and south of Jordan. Data will be transcribed, managed and analysed using interpretative, phenomenological analysis. The Jordanian Ministry of Health and Jordan University Ethics Committee approved the study on Nov. 2019, FEB 2020, respectively. Discussion: The findings of this study will make an original contribution to our knowledge about how stillbirth is experienced by mothers in Jordan. This finding will be useful to health care providers to improve the quality of health care provided to those mothers by adopting individualized and holistic care approach to meet the needs of each mother. Moreover, it will inform public health authorities and policy makers regarding the need for more awareness of stillbirth and a better bereavement care strategy for mothers in hospitals.
\end{abstract}

\section{Keywords}

Stillbirth, Phenomenology, Jordan, Qualitative Research

\section{Introduction}

Across the world, there is a variation in the definition of stillbirth. The world health organization (WHO), defines stillbirth according to the $10^{\text {th }}$ edition of the 
International Classification of Diseases (ICD-10) as "a death that occurs before birth, and classify it according to the time of death as: antepartum stillbirth; before the onset of labour, intrapartum stillbirth; during labour but before birth, and use birth weight equal or more than $500 \mathrm{~g}$, or gestational weeks equal or more than 22 completed weeks, or crown to heel body length equal or more than $25 \mathrm{~cm}$ " for reporting second trimester stillbirth (early fetal death), and use birth weight equal or more than $1000 \mathrm{~g}$, or gestational weeks equal or more than 28 completed weeks, and crown to heel body length equal or more than $35 \mathrm{~cm}$, for reporting third trimester stillbirth (late fetal death), and for international comparison [1] [2]. In Jordan, stillbirth definition is consistent with the world WHO definition of stillbirth, and we use the cut-off point of 22 completed weeks of gestation for reporting early fetal death, and the cut-off point of 28 completed weeks of gestation for reporting late fetal death, regardless the onset of death as antepartum or intrapartum stillbirth.

Throughout the world, there are more than 2.6 million stillbirths that continue to occur every year, ninety eight percent of them occurred in low and middle income countries [3]. In Jordan, from 22.591 babies born at 20 weeks of gestation or later, 261 were stillbirths; 11.6 per 1000 total births born after 20 weeks of gestation, 11.2 per 1000 total births born at 22 weeks of gestation, 10.6 per 1000 total births born 24 weeks of gestation, and 9.0 per 1000 total births born after 28 weeks of gestation [4]. This percentage is higher than many Arab countries in the region such as Kuwait, and Bahrain where the stillbirth rate is the lowest (5.1), (5.5) per 1000 birth, respectively [5]. At the same time, the percentage of stillbirth in Jordan is lower than in other Arab countries, such as Mauritania, Yemen, and Somalia where stillbirth rate is the highest in this region (27.1), (29), (35.5) per 1000 births, respectively [5]. The experience of stillbirth leaves Mothers' suffers from several forms of psychosocial morbidities. The review by Burden et al. [6] shows that mothers after stillbirth suffer from much psychological morbidity, such as: long-term depression, anxiety disorders, post-traumatic stress disorder (PTSD), long-term guilt, and grief. Moreover, several studies show that mothers after stillbirth are at more risk for many social morbidities, such as social isolation, change in their personal relationships with community and wider family members, such as their husband, grandparents, their subsequently born children or siblings, phobia, suicidal thoughts, shame, and severely impaired self-esteem and appraisal. They are blamed for the death of their fetus, and feel stigmatized and rejected through avoidance, divorce and physical abuse [6] [7] [8]. Despite these adverse consequences of stillbirth, it remains neglected public health issue of importance to mother's and wider society. Reducing its burden globally remains slow and insufficient by health policy and programs, in which they do not consider stillbirth in their priorities; in the Millennium Development Goals, stillbirth was missing and still missing in the Sustainable Development Goals [9]. Moreover, globally, there is underreporting for stillbirth cases in vital statistics [10], and health care providers and policy makers even in high income countries like United states of America and United Kingdome un- 
derestimated stillbirth and considered it as less traumatic than the death of a neonate [5] [11] [12]. In Jordan, stillbirth not included in vital statistics reporting system, they only register neonates who live more than four hours after birth and registered it as neonatal deaths, but any neonate who dies before four hours after birth is not registered or registered as stillbirth [13]. Stillbirth is not neglected only from policy maker and health care providers, different society from both high income countries such as United states of America, Australia, New Zealand recently recognized stillbirth as actual loss for parents and recognized their grief, however, several and low-middle income countries such as India, Somalia, Malaysia did not recognize stillbirth as a death of an older child; they did not recognized parents grief, and not recognized the bereavements rituals for stillbirth such as naming, funeral, or mother holding and seeing for the baby [7] [10] [14] [15] [16]. Underestimating and neglecting stillbirth as a meaningful loss will lead to more morbidities for mothers, and that will effect on the wider family system and community as a whole. In Jordan there is a lack of both quantitative and qualitative studies about the burden of stillbirth on mothers and what they need. Consequently, this study aims to explore the meanings attributed by mothers' to their experience of stillbirth in Jordan, and to provide an original contribution to the knowledge in the area of Jordanian mothers' experiences in this field.

\section{Methodology and Methods}

\subsection{Methodology}

This study will adopt a qualitative phenomenological research approach. Qualitative research approach, is more holistic, focuses on the human experience, subjectivity and considers the interrelationship between body, mind, spirit, and environment [17]. Thus, it will provide rich data that help in understanding the unique and subjective experience of mothers who lose their baby and uncover their thoughts, emotions and feelings without being driven by predefined quantitative measures. According to Martin Heidegger philosophical approach, phenomenology aims to understand the attributed meaning of human lived experiences about a phenomenon [18]. In nursing research, phenomenology as a research method is greatly used, since it helps in generating methodical, systematic, critical and inter-subjective knowledge [19]. The concept of "Being in the world" Dasein in relation to Heideggerian phenomenology and Heidegger Analysis of "Being" [20], will be applied to understand the experience and health care need of mother's after stillbirth.

\subsection{Methods}

\section{Study design}

For the purpose of this study, an interpretative phenomenological analysis (IPA), which has its theoretical foundations in phenomenology, will be used. (IPA) is a qualitative methodology which is focused on how individuals reflect 
on and make sense of a significant life-experience and show how this subjective experience is only accessible through interpretation [21]. (IPA) greatly used in healthcare research as it helps the researchers to rigorously investigate the psychosocial phenomena with possible impact on patient care [18].

\section{Setting of the study}

The study will be conducted at three governmental hospitals: Al-base hospital, it is the largest public and referral hospital in Amman at middle of the Jordan; Princess Badia'a Hospital, it is the largest referral and teaching hospital in the north of Jordan specialized in providing family planning services, treatment and follow-up of gynaecological diseases and obstetric surgery; and Ma'an governmental hospital, it is one of the largest hospital in south of Jordan.

\section{Sampling methods}

This study is guided by Heideggerian interpretive phenomenology. Thus, a purposeful quota sampling strategy will be used in this study to achieve maximum variation. Purposeful sampling is commonly used in qualitative research [22]. It involves selecting research participants according to the needs of the study and their particular knowledge of the phenomenon; to provide rich data and help the researcher to gain in-depth understanding of the phenomena under study [23]. Following guidelines of qualitative research sample size is determined based on the quality and the richness of themes or patterns of meaning emerging from the data [24], this is called the theoretical or thematic saturation; in which the researcher will continue conducting the interviews and collecting data until repetition and redundancy occurs during data analysis [17]. In this study, the target population will be mothers who lived the experience of stillbirth. The accessible population will be mother who lived the experience of stillbirth and meet the eligibility criteria. The inclusion and exclusion criteria for study participants are provided below:

The inclusion criteria will be: Mother with a stillborn baby, at or more than twenty-eight gestational weeks (Late fetal death), mothers who experienced a stillbirth within the past one year from the time of diagnosis, and mothers must be willing to share their thoughts about the experiences, and can articulate their experiences.

The exclusion criteria will be: Mother who previously experienced multiple perinatal losses (stillbirth), and mother who had multiple pregnancies with a surviving fetus.

\section{Access to the participants}

The childbirth ward in each hospital will be visited and the head nurses will be asked to facilitate the recruitment of potential participants by retrospective analysis of medical health records and electronic medical health records for mothers diagnosed as a previous stillbirth case and met the eligibility criteria of the study, after that the researchers will follow the recruitment strategy for selecting study participants, Figure 1.

\section{Data collection procedures}




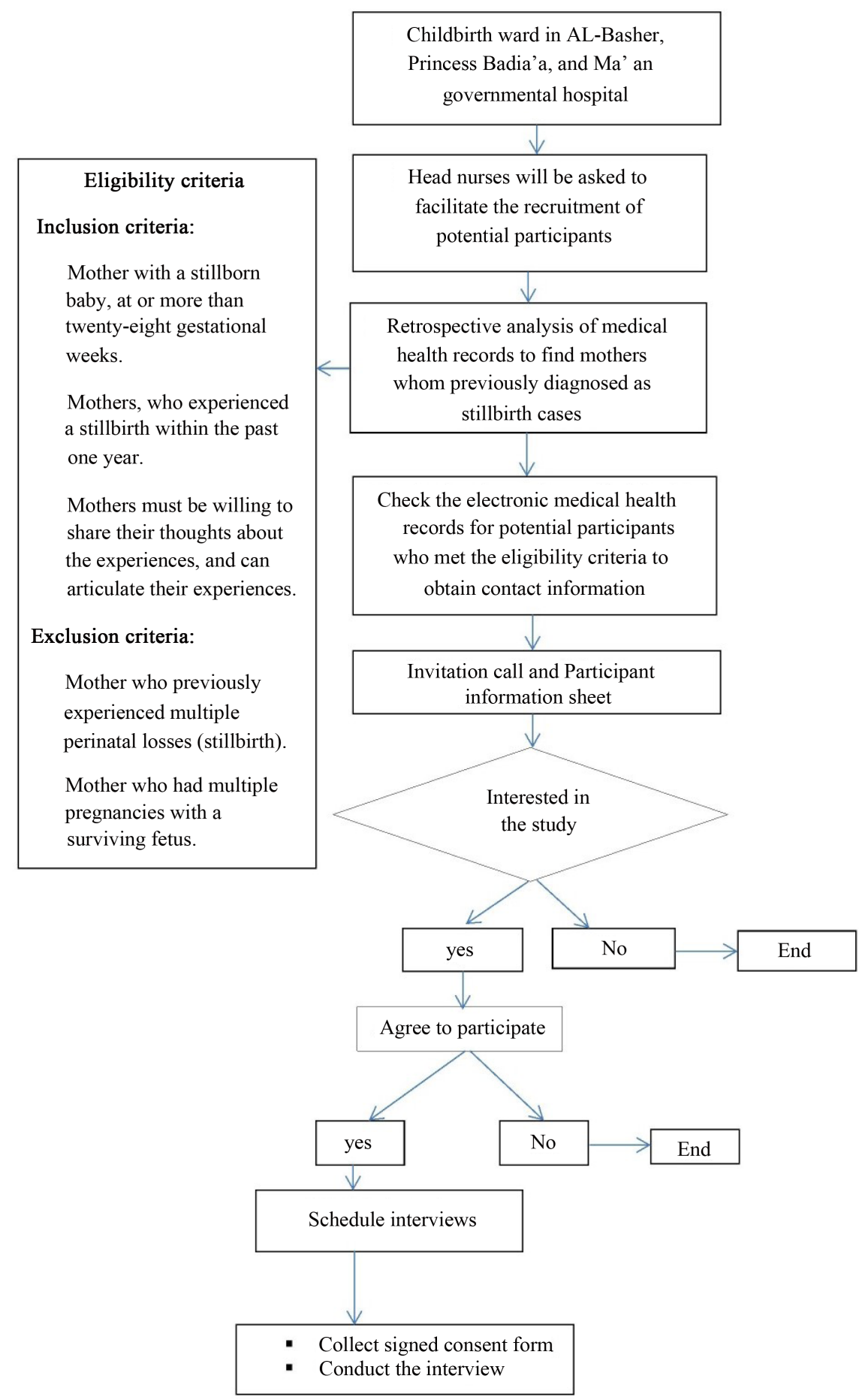

Figure 1. Recruitment strategy for selecting study participants.

Each mother who agree to participate in the study will be requested to sign consent forms before the interview begins, in which they will agree that the interview can be audio-recorded and written notes can be taken. A single individualized semi-structured interviews will be conducted at the preferred place of the participant, Semi-structured interviews will be used to help the researcher to collect subjective data that reflect the perspective of mothers about stillbirth ex- 
perience, The interview guide help the researcher to make sure that the important areas to understand the experience from the perspective mothers who live it is covered, and at the same time the flexibility of semi-structured interview still allowing the participants to introduce new issues that the researcher may not previously thought about it [25] [26]. That will lead to more reflexive and mutual creation of knowledge between researcher and participants and achieve the goal of interpretive phenomenology. Interviews will be last between 60 to 90 minutes, Interviews will be audio recorded and non-verbal responses will be documented as field notes. Before the interview began the researcher will make sure ensure that a mother feels comfortable to be interviewed and after the interview emotional support will be offered, if needed. Each interview then will be transcribed from an audio recording into written form in Arabic language, and no identifying characteristic for the participants will be included in the transcription.

\section{Ethical considerations}

Ethical approval is given by the Jordanian Ministry Of Health and Jordan University Ethics Committee (Moh/REC/2020/30) and (PDs.19.2), respectively. Participants will be asked to sign a consent form to indicate their willingness to participate. Voluntary participation and the right to ask any questions and to right to withdraw from the study at any time will be emphasized during the data collection.

\section{Data analysis}

Transcripts of the interviews will be analysed manually by using an interpretative phenomenological approach as outlined by Smith et al. [27]. The analysis included the following five steps: firstly: familiarization for each interview transcript through read and re-read in conjunction with the revision of researcher field note. Secondly, the initial noting in which the researchers will independently identify the emerging themes from each transcripts following a line-by-line analysis of the interview. Thirdly, developing emergent themes, in this step the researcher will focus on the specific key words or phrases that frequently appeared throughout the interview transcript emerging themes will be recorded as phrases or sentences throughout each individual transcript; to capture the essential quality of data. In a fourth step which is searching for consistencies across emergent themes, the researcher will initiate clustering of themes according to their interconnections similar themes will be grouped together, throughout the process, as the researcher will interpret the data, emerging themes will be defined and redefined with the integration of collective cases, then a list of superordinate and subordinate themes will be created. Finally, a summary table noting each participant's contribution to the themes and supported by extracts from the transcripts will be created.

\section{Discussion}

This study protocol represents the first study to investigate thoroughly the phenomenon of stillbirth from Jordanian mother's perspective. The lack of evidence 
about how this phenomenon is lived by Arabic mothers in Arabic culture underlines the importance of conducting this study to generate new evidence and knowledge. The findings of this qualitative study will be context - specific and not generalizable to other settings or populations. However, it is hoped that the findings could provide rich and detailed understanding into stillbirth phenomena and mother's needs, this understanding could have significant implications for improvement of future clinical practice and policy regarding the health care provided to bereaved mothers, by adopting a holistic and individualized approach of nursing and health care based on the needs and values that bereaved mothers have after stillbirth. The American Holistic Nurses' Association (AHNA) defines holistic nursing as "All nursing practice that has healing the whole person as its goal" [28]. Thus, holistic nurses are those who recognize and treat each person differently and consider all the physical, mental, spiritual, cultural, socioeconomic and environmental aspects when delivering care; because all these aspects are interconnected and fit together to form the uniqueness of each person [28]. Adopting the individualized- holistic care approach for mothers experiencing stillbirth will contributes to a high standard of healthcare, as well as can leads to better experience, normal grieving process and more positive outcomes for bereaved mothers [29]. It is also expected that the development of this protocol could provide a base of evidence for further research studies of stillbirth combined with quantitative methods.

\section{Conclusion}

Mothers' loss of their baby in late pregnancy period is associated with multi psychosocial and physical morbidities. In Arabic culture, the phenomena of stillbirth and how it affects on mothers' is not clearly understood. This study protocol represents the first study to deeply investigate the phenomenon of stillbirth from Jordanian mothers' perspectives, and the lack of evidence in this specific context underlines the importance of conducting this study to fill the knowledge gab and to generate new empirical knowledge in this field. The findings of qualitative research are context-specific and not generalizable to other settings or populations. However, it is hoped that the findings could offer a rich and detailed insight into the phenomenon of stillbirth and could be beneficial to the development of future guidelines and policies to improve the clinical practice toward those mothers' in Jordanian hospitals.

\section{Availability of Data and Materials}

The data described in this paper are referring to this study protocol only and there are no raw data reported. The datasets will be collected and analysed and can be available from the corresponding author on request.

\section{Acknowledgements}

The authors' would like to acknowledge the financial support of the AL-Hussein 
Bin Talal University for awarding the corresponding author a postgraduate scholarship.

\section{Conflicts of Interest}

All authors declare that there are no conflicts of interest associated with this study.

\section{References}

[1] WHO (2016) The WHO Application of ICD-10 to Deaths during the Perinatal Period. World Health Organization, 1-88.

[2] Frøen, J.F., et al. (2009) Making Stillbirths Count, Making Numbers Talk-Issues in Data Collection for Stillbirths. BMC Pregnancy Childbirth, 9, 1-17. https://doi.org/10.1186/1471-2393-9-58

[3] Blencowe, H., et al. (2016) National, Regional, and Worldwide Estimates of Stillbirth Rates in 2015, with Trends from 2000: A Systematic Analysis. The Lancet Global Health, 4, e98-e108. https://doi.org/10.1016/S2214-109X(15)00275-2

[4] Khader, Y., Batieha, A.K. and Hamadneh, S. (2018) Stillbirths in Jordan: Rate, Causes, and Preventability. The Journal of Maternal-Fetal \& Neonatal Medicine, 33, 1307-1314. https://doi.org/10.1080/14767058.2018.1517326

[5] WHO (2015) Stillbirths Estimates by Country. Global Health Observatory Data Repository. https://apps.who.int/gho/data/view.main.GSWCAH06v

[6] Burden, C., et al. (2016) From Grief, Guilt Pain and Stigma to Hope and Pride: A Systematic Review and Meta-Analysis of Mixed-Method Research of the Psychosocial Impact of Stillbirth. BMC Pregnancy Childbirth, 16, 9. https://doi.org/10.1186/s12884-016-0800-8

[7] Heazell, A.E.P., et al. (2016) Stillbirths: Economic and Psychosocial Consequences. The Lancet, 387, 604-616. https://doi.org/10.1016/S0140-6736(15)00836-3

[8] Hill, P.W., Cacciatore, J., Shreffler, K.M. and Kayla, M. (2016) The Loss of Self: The Effect of Miscarriage, Stillbirth, and Child Death on Maternal Self-Esteem Abstract. 1187.

[9] Froen, J.F., et al. (2011) Stillbirths: Why They Matter. The Lancet, 377, 1353-1366. https://doi.org/10.1016/S0140-6736(10)62232-5

[10] Lawn, J.E. and Kinney, M. (2011) An Executive Summary for The Lancet's Series.

[11] Kelley, M.C. and Trinidad, S.B. (2012) Silent Loss and the Clinical Encounter: Parents' and Physicians' Experiences of Stillbirth: A Qualitative Analysis. BMC Pregnancy Childbirth, 12, 137. https://doi.org/10.1186/1471-2393-12-137

[12] Downe, S., Schmidt, E., Kingdon, C. and Heazell, A.E.P. (2013) Bereaved Parents' Experience of Stillbirth in UK Hospitals: A Qualitative Interview Study. BMJ Open, 3, 2. https://doi.org/10.1136/bmjopen-2012-002237

[13] Khader, Y., Al-sheyab, N., Alyahya, M. and Batieha, A. (2018) Registration, Documentation, and Auditing of Stillbirths and Neonatal Deaths in Jordan from Healthcare Professionals' Perspectives: Reality, Challenges and Suggestions. The Journal of Maternal-Fetal \& Neonatal Medicine, 1-11. https://doi.org/10.1080/14767058.2018.1531120

[14] Flenady, V., et al. (2016) Stillbirths: Recall to Action in High-Income Countries. The Lancet, 387, 691-702. https://doi.org/10.1016/S0140-6736(15)01020-X

[15] Osman, H.M., Egal, J.A., Kiruja, J., Osman, F., Byrskog, U. and Erlandsson, K. 
(2017) Women's Experiences of Stillbirth in Somaliland: A Phenomenological Description. Sexual \& Reproductive HealthCare, 11, 107-111. https://doi.org/10.1016/j.srhc.2016.12.002

[16] Sutan, R. and Miskam, H.M. (2012) Psychosocial Impact of Perinatal Loss among Muslim Women. BMC Women's Health, 12, 2-9. https://doi.org/10.1186/1472-6874-12-15

[17] Creswell, J.W. and Poth, C.N. (2017) Qualitative Inquiry and Research Design: Choosing among Five Approaches. Sage Publications, New York.

[18] Lopez, K.A. and Willis, D.G. (2004) Descriptive versus Interpretive Phenomenology: Their Contributions to Nursing Knowledge. Qualitative Health Research, 14, 726-735. https://doi.org/10.1177/1049732304263638

[19] Applebaum (2012) Key Ideas in Phenomenology: The Natural Attitude. https://www.saybrook.edu/unbound/phenomenology/

[20] Heidegger, M. (1962) Being and Time. Blackwell, Oxford.

[21] Horrigan-Kelly, M., Millar, M. and Dowling, M. (2016) Understanding the Key Tenets of Heidegger's Philosophy for Interpretive Phenomenological Research. International Journal of Qualitative Methods, 15, 1-8. https://doi.org/10.1177/1609406916680634

[22] Mohajan, H. (2018) Qualitative Research Methodology in Social Sciences and Related Subjects. Economic Policy, 7, 23-48. https://doi.org/10.26458/jedep.v7i1.571

[23] Braun, V. and Clarke, V. (2013) Successful Qualitative Research: A Practical Guide for Beginners. Sage Publications, New York.

[24] van Manen, M. (1990) Researching Lived Experience: Human Science for an Action Sensitive Pedagogy. State University of New York Press, Albany, NY.

[25] Guerrero-Castañeda, R.F., Ojeda-Vargas, M.G. and Menezes, T.M.O. (2017) Characteristics of the Phenomenological Interview in Nursing Research. Revista Gaúcha de Enfermagem, 38, e67458. https://doi.org/10.1590/1983-1447.2017.02.67458

[26] Bolderston, A. (2012) Conducting a Research Interview. J. Med. Imaging Radiat. Sci., 43, 66-76. https://doi.org/10.1016/j.jmir.2011.12.002

[27] Tindall, L., Smith, J.A., Flower, P. and Larkin, M. (2009) Interpretative Phenomenological Analysis: Theory, Method and Research. Qualitative Research in Psychology, 6, 346-347. https://doi.org/10.1080/14780880903340091

[28] American Holistic Nurses Association (2018) What Is Holistic Nursing? http://www.ahna.org/About-Us/What-is-Holistic-Nursing

[29] Ellis, A., et al. (2016) Systematic Review to Understand and Improve Care after Stillbirth: A Review of Parents' and Healthcare Professionals' Experiences. BMC Pregnancy Childbirth, 16, 1-19. https://doi.org/10.1186/s12884-016-0806-2 